\title{
Vocación del servicio en Colombia y nuevas formas de colonialismo en la lógica managerial $^{*}$
}

\author{
Vocation of civil servant in Colombia and new ways of colonialism in the managerial logic \\ Vocação do serviço na Colômbia e novas formas de colonialismo na lógica managerial
}

Oscar Andrés López Cortés a
Universidad Libre, Colombia
lopez-oscar@javeriana.edu.co
DOI: https://doi.org/10.11144/Javeriana.cao32-58.vscn Redalyc: http://www.redalyc.org/articulo.oa $? \mathrm{id}=20560207007$

Fecha de recepción: 27/09/2018

Fecha de aprobación: 29/03/2019

Fecha de publicación: 30/06/2019

\section{Resumen:}

Este artículo presenta los resultados de una investigación etnográfica y de análisis documental sobre la vocación del servicio en el sector público. La hipótesis es que la vocación del servicio entró en la gestión pública nacional como parte de un conjunto de reformas administrativas orientadas por la lógica managerial, adoptadas por los gobiernos de Colombia para atender las exigencias de los organismos internacionales, configurándose así una nueva forma de colonialidad epistémica. Se concluye que en las prácticas y los discursos institucionales que definen y prescriben la vocación del servicio, esta se asume como un atributo natural de las personas que laboran en la administración pública y que se puede incentivar sin importar las condiciones objetivas de trabajo.

Códigos JEL: J78, K31, M12, M54

Palabras clave: vocación, reformas administrativas, gestión pública, lógica managerial.

\section{Abstract:}

This paper exposes the results of ethnographic and documental analysis research. The hypothesis argues that the vocation of civil servant came into the National public management as part of administrative reforms set guided by the managerial logic, adopted by Colombian governments to attend the international organizations requirements, thus configuring a new way of epistemic colonialism. The conclusion is that in the institutional practices and speeches that define and prescribe the service vocation this is assumed as a normal attribute of the civil servants, and thus, service vocation could be incentivized no mater the objective conditions of labor.

JEL Codes: J78, K31, M12, M54

Keywords: Vocation, administrative reforms, public management, managerial logic.

\section{Resumo:}

Este artigo apresenta os resultados de uma pesquisa etnográfica e de análise documental sobre a vocação do serviço no setor público. A hipótese é que a vocação do serviço ingressou na gestão pública nacional como parte de um conjunto de reformas administrativas orientadas pela lógica managerial, adotadas pelos governos da Colômbia para atender às exigências das organizações internacionais, configurando-se assim uma nova forma de colonialidade epistêmica. Conclui-se que nas práticas e discursos institucionais que definem e prescrevem a vocação do serviço, esta é assumida como um atributo natural das pessoas que trabalham na administração pública e que pode ser estimulada independentemente das condições objetivas de trabalho.

Códigos JEL: J78, K31, M12, M54

Palavras-chave: vocação, reformas administrativas, gestão pública, lógica managerial.

"Volvimos a nuestro hogar, estos Reinos

Pero no se siente cómodo aqui,

Con un pueblo ajeno aferrándose a sus dioses.

Me alegraría de otra muerte."

(Poema El viaje de los Magos de T. S. Eliot)

Notas de autor:

${ }^{a}$ Autor de correspondencia. Correo electrónico: lopez-oscar@javeriana.edu.co 
Me alegraría de otra muerte (No Longer at Ease, 1960), novela de Chinua Achebe, describe la situación de Obi Okonkwo, quien tras estudiar en Inglaterra regresa a su natal Nigeria para desempeñarse como empleado público. La novela describe el peso de la relación colonial de Europa sobre África durante el período de descolonización de la década del 1950. No obstante, la descolonización es puesta en tela de juicio por Achebe, quien muestra cómo se perpetuó esa relación, no sólo en las estructuras jurídicas y políticas de las naciones que allí afloraron, sino en las subjetividades como las del imaginario Obi Okonkwo.

La continuidad del colonialismo se manifiesta en la agenda de reformas que los estados europeos y norteamericanos imponen en África y América Latina. Entre los años 2010 y 2017, una de las prioridades del gobierno colombiano fue conseguir la admisión del país en la Organización para la Cooperación y el Desarrollo Económico -OCDE-; entre los requisitos que debía cumplir para ello estaba modernizar el Estado, lo que requería una transformación legal de la estructura de la administración pública. Pero con otras prioridades en la agenda política, el gobierno colombiano adoptó por vía de decretos algunas medidas para transformar la administración, entre las cuales se encuentra la generación de una cultura de la vocación del servicio.

Este período de cambios en la administración pública en Colombia está marcado por nuevas formas de intervención colonial, en particular las impuestas por la OCDE. El objetivo principal de este artículo es identificar la manera como se instala el discurso de la vocación del servicio en las entidades públicas donde se realizó el trabajo de campo etnográfico, para lo cual se definió la siguiente hipótesis: la vocación del servicio ha entrado en la gestión pública como parte de un conjunto de reformas administrativas orientadas por la lógica managerial que han sido adoptadas por los gobiernos de Colombia desde finales de la primera década del siglo, para atender exigencias de los organismos internacionales, configurándose así una nueva forma de colonialidad epistémica.

Este artículo se divide en cuatro partes: el método empleado en la investigación, el contexto político de las reformas en la administración pública durante el periodo analizado, el concepto de vocación del servicio, y finalmente, con apoyo en los estudios críticos del trabajo, el análisis de las prácticas institucionales sobre la vocación del servicio en el marco de las reformas promovidas por la OCDE en Colombia.

\section{El método empleado}

Entre 2012 y 2015 hice parte de la práctica “Mundo del Trabajo, Cultura y Subjetividad” dentro del cual se desarrolló un proyecto intervención-investigación entre el Departamento de Psicología del Trabajo y las Organizaciones de la Facultad de Psicología de la Pontificia Universidad Javeriana y el Departamento Administrativo de la Función Pública -DAFP-. Un equipo de profesores orientábamos la práctica de estudiantes de últimos semestres de psicología realizada en el DAFP, al cabo de la cual entregaban a la Entidad una lectura cultural al cabo de la cual se entregaba un informe (Pulido, 2008).

Durante el proyecto la Entidad solicitó una indagación particular sobre la vocación del servicio en el empleo público. El requerimiento surgió porque la OCDE había recomendado al gobierno colombiano modernizar la administración pública, y la Entidad consideró necesario reforzar la vocación del servicio. Procedimos entonces a diseñar un microproyecto de investigación que atendiera al requerimiento hecho por el DAFP. Para ello, revisamos literatura, leyes, decretos, sentencias, directrices internas de la función pública, resoluciones administrativas, documentos Conpes, entre otros textos que abordaran el concepto de vocación del servicio. Adicionalmente las estudiantes entrevistaron bajo la coordinación del equipo de profesores a 
129 funcionarios de distintas entidades del poder ejecutivo (ministerios y departamentos administrativos) ubicadas en Bogotá.

Para analizar el material recolectado en las entrevistas, la observación participante y los documentos, tomamos elementos del análisis crítico de discurso y de la etnografía institucional (Das \& Poole, 2008). Combinamos estas herramientas luego de percibir que la lectura de los documentos no bastaba para dar cuenta de la compleja red de relaciones a través de las cuales seentronizaba la cultura managerial en el empleo público.

En cuanto al análisis crítico de discurso es importante recordar la manera como Ruth Wodak define este tipo de narrativa: "complejo conjunto de actos lingüísticos simultáneos y secuencialmente interrelacionados, actos que se manifiestan a lo largo y ancho de los ámbitos sociales de acción como muestras semióticas (orales o escritas y temáticamente interrelacionadas) y muy frecuentemente como «textos»." (Wodak, 2003 p. 105). Los relatos de los entrevistados revelan las condiciones de emergencia del discurso. Así, el discurso no puede ser tomado como un mero repertorio lingüístico de términos dispuestos para la interpretación, sino como aquello por lo cual se lucha y, simultáneamente, es el medio a través del cual se lucha (Foucault, 2010). Los discursos se advierten a través de las prácticas mediante las cuales las personas ejercen relaciones de poder y son simultáneamente sujetos (Foucault, 2000) del poder en los ámbitos concretos donde laboran. En este sentido, el hilo conductor de la investigación es un tipo de analítica del poder que busca:

no ir del discurso hacia su núcleo interior y oculto, hacia el corazón de un pensamiento o de una significación que se manifiesta en él; sino, a partir del discurso mismo, de su aparición y de su regularidad, ir hacia sus condiciones externas de posibilidad, hacia lo que da motivo a la serie aleatoria de esos acontecimientos y que fija los límites. (Foucault, 2010, p. 53)

La observación y el análisis del material recayeron tanto sobre las interpretaciones de las estudiantes en práctica vertidas en los diarios de campo, como sobre los relatos obtenidos a través de entrevistas. La observación de ambos materiales permite entender la manera como la psicología, en tanto discurso científico, interviene en la construcción de la subjetividad del funcionario público, como se señalará más adelante. Las interpretaciones de las estudiantes en práctica ubicadas en el contexto de las entidades públicas son discursos susceptibles de ser analizados como parte de la experiencia etnográfica.

En ese sentido, no buscamos determinar la coherencia ni la validez técnica de los relatos y los documentos que prescriben la vocación de servicio conforme a algún parámetro legal, organizacional, psicológico o administrativo, sino analizar los discursos y las prácticas que constituyen la subjetividad del servidor público en el contexto de las reformas estatales de los últimos años, impulsadas por la intervención de organismos internacionales como la OCDE. La reconstrucción de ese contexto constituye el objetivo del siguiente apartado, y es lo que permitirá comprender cómo surgió la política que busca promover la vocación del servicio.

\section{El contexto de las reformas a la administración pública}

Una de las prioridades de los gobiernos de Juan Manuel Santos, en el plano de las relaciones internacionales, fue lograr que la OCDE admitiera a Colombia como miembro de esa organización. Entre los requisitos exigidos por la OCDE estaba modernizar el Estado, lo que requería cambios estructurales y el desarrollo de prácticas de "buen gobierno" que condujeran a una administración pública orientada por los valores de la eficacia y la rentabilidad propias del management.

Sin embargo, las medidas que buscaban identificar la gestión pública con los valores de la administración de empresas comenzaron con el gobierno de Uribe Vélez. Su administración expidió el Decreto 1601 de 2005, a través del cual se adoptó el modelo para la evaluación de competencias gerenciales y para la provisión de empleos de libre nombramiento y remoción, en el cual se promueve una mirada empresarial de lo público. En 2006, el DAFP realizó en Medellín el Congreso de Competencias Laborales y Cuadros Funcionales, y en 2009, en Cartagena se celebró el Congreso Internacional por una Gestión Pública Competitiva e Innovadora. 
En ambos congresos se desarrollaron los temas que más tarde se impondrían en las reformas: "gestión del conocimiento, las competencias laborales, la gerencia pública, la calidad, el liderazgo, el control social y la rendición de cuentas y la planeación estratégica." (Rodríguez \& Grillo, 2010, p. 353). Rodríguez, entonces directora del DAFP, Grillo, quien también ha dirigido esa entidad, celebraban en el documento de 2010 la adopción en la gestión pública de la lógica de la empresa privada. En esa misma época, entre 2006 y 2009 , el DAFP desarrolló la política de "gerencia pública", comenzando así a utilizar un lenguaje que aproximaba lo público a las teorías del management.

En el capítulo titulado "Consolidar un Estado Eficiente y Transparente y un Modelo de Intervención Económica Óptimo” incluido en el documento Visión Colombia II Centenario: 2019, se adopta un modelo de gestión de empleo público basado en la medición por resultados propia de la empresa privada:

El modelo postburocrático que deberá imperar en la estructura administrativa colombiana debe caracterizarse por el gobierno de la información, la innovación administrativa, la prestación de servicios hechos a la medida; la competencia global y "administrada", la gestión por resultados, la flexibilidad laboral en función de los resultados y la ejecución de políticas de mejoramiento continuo. (Cepal, s.f., p. 332)

Esta meta planteada en el gobierno Uribe continuó durante el Gobierno Santos, y fundamentó las acciones tomadas entre 2011 y 2017 para buscar la entrada de Colombia a la OCDE. Con tal objetivo, se impulsó una reforma jurídica a la estructura del empleo público, la cual provocó la creación de las agencias nacionales. En principio, tal reforma debía debatirse en el Congreso de la República, en tanto fijar la estructura del Estado es una competencia que la Constitución Política asignó al poder legislativo. Para saltarse al legislador, el gobierno acudió a las facultades excepcionales otorgadas en los planes de desarrollo. Así, se crearon entre 2011 y 2017 catorce agencias nacionales.

Estas agencias no existían en la Ley 489 de 1998 que regula la estructura de la administración pública, por el contrario, fueron incluidas en la reforma administrativa de "buen gobierno" propuesta en el primer Plan Nacional de Desarrollo de la administración de Juan Manuel Santos (Garrido, 2017). Las agencias tienen un régimen salarial especial excluido de las normas previstas para los demás servidores públicos. El Ejecutivo argumentó que tal excepción se justificaba en tanto quienes integran las agencias son personas de elevada calificación que pueden ser atraídos por el sector privado, y para evitar esa fuga, era necesario otorgarles un régimen salarial especial. Adicionalmente, las agencias nacionales gozan de autonomía administrativa, financiera, técnica y presupuestal (Sarria, 2015), no obstante lo cual están adscritas a los ministerios del ramo al que pertenecen según la actividad que ejecutan.

Pero la creación de agencias especializadas no fue la única estrategia implementada para "modernizar el Estado" y desarrollar la política de "buen gobierno". La segunda estrategia se estructuró a partir de varias acciones que no implicaban reformas al marco jurídico del empleo público y la estructura administrativa. La primera acción consistió en atraer a los cargos directivos de la administración pública a personas que se habían desempeñado en el sector privado, con el argumento de que serían capaces de proyectar en las entidades estatales los valores que hacían productivas a las empresas privadas, como la innovación, el liderazgo y la creatividad. Esta acción se llevó a cabo en diferentes entidades del sector público, comenzando por el DAFP. Como resultó evidente a través de la observación participante, las personas encargadas de dependencias claves de esa entidad durante esos años, venían del sector privado y no tenía experiencia en el sector público. La segunda acción se ocupaba de generar una cultura de la vocación del servicio, y fue la que ocupó la atención

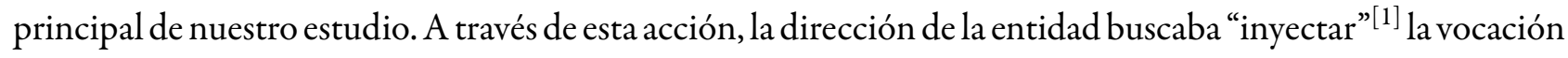
del servicio a los funcionarios públicos.

La primera inquietud que surgió entonces fue cómo se puede definir conceptualmente la "vocación del servicio”, para lo cual fue necesario revisar la literatura al respecto. Los resultados de esa revisión documental se condensan en el siguiente apartado. 


\section{El concepto de vocación del servicio en la literatura}

En Colombia, la primera alusión al concepto vocación de servicio en un documento institucional aparece en Cartillas de Administración Pública. Plan nacional de formación y capacitación de empleados públicos para el desarrollo de competencias. Este documento fue publicado en 2010 por el DAFP y la Escuela Superior de Administración Pública -ESAP-, principal institución educativa oficial que forma en Colombia a los administradores públicos. Ese documento emplea el concepto para señalar que, junto al mérito, la capacidad, la eficacia en el desempeño de la función, la responsabilidad, la honestidad y la adhesión a los principios y valores de la democracia, la vocación de servicio es un atributo más que debe tener todo servidor público. La razón atiende a un criterio normativo: porque así lo establece la Carta Iberoamericana de la Función Pública CIFP-. Como se puede apreciar, desde un principio la vocación de servicio apareció como una prescripción de la norma jurídica sobre el sujeto. Sin embargo, este origen se ha olvidado con el tiempo y se ha asumido que la vocación del servicio debe ser un rasgo inherente del buen servidor público, borrando así el origen normativo del concepto y naturalizando su función. Esto último se evidencia en el producto de una consultoría que el DAFP adjudicó a la Escuela de Gobierno Alberto Lleras Camargo de la Universidad de los Andes, a través de la cual se publicó el texto Gestión estratégica del talento humano en el sector público: estado del arte, diagnóstico y recomendaciones para el caso colombiano, en el cual se recomienda exaltar la vocación de servicio como propuesta de cambio dentro de la gestión estratégica del talento que no requiere reformas legales. En tal perspectiva, la vocación del servicio aparece como un atributo natural de las personas que laboran para la administración pública, sin que las condiciones objetivas de trabajo sean factores determinantes.

El documento CONPES No 3785 de 2013 definió la vocación de servicio como un principio rector referido "a una concepción del empleo y del talento humano al servicio del Estado y de la ciudadanía" (DNP, 2013 , p. 9). En ese mismo documento, la vocación de servicio se considera uno de los ejes del Buen Gobierno. La definición, aunque clara, es general, lo que exige rastrear las posibles dimensiones de la vocación del servicio. Esto supondría, en primer lugar, rastrear el concepto en la literatura especializada de la administración pública y ver luego cómo esta construcción semántica es apropiada por los funcionarios en sus prácticas discursivas, mediante las cuales el sentido de la vocación es construido socialmente, dotado de significado y desarrollado como parte de la experiencia cotidiana.

Al consultar los documentos disponibles en aquella época a partir de bases de datos propias del campo de la administración pública, resultaba notorio el vacío conceptual existente a propósito de la vocación de servicio. Se carecía entonces de una definición al respecto, tanto en el campo de los documentos de política, pero aun más, en la academia, salvo contadas excepciones, como Gómez y Arango (2012). Hoy algunos trabajos se han ocupado de señalar la vocación de servicio como un rasgo que debe caracterizar al buen funcionario público (Ospina \& Hofmann-Pinilla, 2015); como un elemento del talento humano relevante para la planeación estratégica o como criterio orientador de la función pública (Sanabria, 2015). Sin embargo, en ninguno de estos documentos se define qué es la vocación del servicio.

Ante tal vacío, la búsqueda se amplió a otros campos, y el que resultó más fructífero fue el de la administración de empresas. Allí, el concepto de vocación del servicio es usualmente relacionado con la ética, el compromiso, la honestidad, el sacrificio y otra serie de valores propios de un "buen funcionario". Se trata de un concepto dúctil según las necesidades y los imperativos morales vigentes, impregnado del lenguaje del management, no de la axiología ni de la administración pública.

En la administración de empresas la vocación de servicio se asocia a las cualidades y comportamientos propios de los "buenos trabajadores". Barrascout, por ejemplo, identifica la vocación de servicio como elemento del "espíritu de servicio" que debe guiar a la persona; el trabajador con vocación es definido como alguien solícito y de buen ánimo para hacer las cosas y ayudar a quien lo necesite. Para el autor "gente con vocación de servicio es el recurso que toda empresa necesita para progresar.” (Barrascout, 2012, p. 80). 
La literatura destaca la vocación de servicio como hábito y elemento cultural que deben fomentar las organizaciones incentivando las acciones propias de dicho hábito. Esa misma literatura señala la autosatisfacción o el placer de saber que con sus acciones el sujeto ha servido a otros, como el más importante reconocimiento que la organización debe resaltar del trabajador con vocación de servicio (Lira, 2009, p. 35).

La connotación religiosa de la vocación como atención al llamado de fe con el que Weber (1994) asociaba la expresión berüf, parece aun estar presente, pero de manera instrumental, pues se promueve como la recompensa que el trabajador obtiene por la vocación. En cambio, para la organización la vocación de servicio de sus trabajadores permite obtener beneficios materiales, no mera autosatisfacción, sino aumento de la productividad, fidelidad de los clientes, incremento de utilidades, buena imagen corporativa y eficiencia en la gestión.

La vocación de servicio no es exclusiva de las personas vinculadas por una relación laboral de subordinación. En entornos productivos caracterizados por el trabajo independiente o por cuenta propia, Braulio Pareja señala que la vocación de servicio ha sido destacada como atributo propio de los nuevos gerentes, para quienes esta, junto a "los valores, la proactividad, y la capacidad para liderar proyectos y movilizar personas" (Pareja, s.f.. p. 14), son condiciones esenciales para el éxito y la consolidación de la marca personal.

La vocación de servicio se identifica como un pilar del servicio al cliente y una virtud propia de cualquier trabajador contemporáneo, independiente de si se trata del clásico trabajador fordista, el nuevo empleado flexibilizado o el independiente "empresario de sí mismo" (Costhek, 2018; Foucault, 2006; Muñoz, 2006; López, 2010; Peña, 2012; Pierbattisti, 2008; Agüero, 2010; Aubert \& Gaulejac, 1993). Virtud y pilar que deben desarrollarse si se desea alcanzar el éxito en el mundo laboral.

Como sucede en otros campos, el sector público ha recibido la influencia de métodos, teorías, conceptos y prácticas originadas en la administración de empresas. Este proceso, al que diversos autores (Chandler, Barry, \& Clark, 2002; Dall, 2009) han denominado como el Nuevo Management Público -NMP-, y que se deriva de lo que otros (Thomas \& Davies, 2005; Spink, 2009; Ascorra, 2010; Pierbattisti, 2012), siguiendo lo propuesto por Parker (2002), llaman "lógica managerial", ha reconfigurado el Estado mediante la implantación de la lógica propia de las empresas privadas.

$\mathrm{Al}$ revisar la literatura de la administración de empresas resultó evidente la manera como el NMP es producto de una lógica de intervención colonial a través de la cual el discurso promovido por las agencias internacionales se implanta en las reformas estatales. Estas reformas no operan únicamente a través de cambios legales, los cuales son notorios a simple vista, sino en los pequeños acontecimientos cotidianos, mucho más difíciles de percibir. Como lo han estudiado los autores referenciados, es la lógica managerial la que reconfigura el Estado, y no las propuestas de origen ciudadano, mucho menos las propuestas de las organizaciones sindicales del sector público. Las directrices externas formuladas en los Estados que gobiernan las instituciones internacionales resultan más importantes que las necesidades y condiciones concretas de sus propias entidades y de sus propios empleados.

En la medida que quienes gobiernan asumen que la administración de empresas es un saber cuyas prácticas son más eficaces, se le reconoce a esa disciplina un estatuto científico superior al cual debe ceñirse la administración pública. En esa lógica, la gestión del Estado debería parecerse más al de una empresa privada, porque se presume que eso ha funcionado en los países desarrollados. Esa mirada reproduce una forma de intervención colonial epistémica, a través de la cual los pueblos en vías de desarrollo, periféricos o del tercer mundo, no podemos auto-reconocernos en nuestras particularidades y pensar por nosotros mismos, sino que debemos ceñirnos a las imposiciones, disfrazadas bajo eufemismos como recomendaciones, sugerencias, orientaciones, y demás directrices que imparten organismos como la OCDE.

Las maneras como se instala la lógica de la empresa privada en el sector público para el caso colombiano, están estrechamente relacionadas con elementos del contexto histórico legalista determinado por la tradición jurídica colombiana, a la que, con apoyo en Marta Zambrano (2000), se puede denominar como la judicialización de la vida cotidiana en la entidad pública. 
La forma como ocurre esta judicialización de la vida, se evidencia en lo planteado por Zambrano (2000) a propósito del efecto de la escritura legal desde la Colonia. Según ella, entre los siglos XVI y XVIII, la mayoría de los pobladores del Nuevo Reino de Granada, incluyendo colonizados, colonizadores pobres y mujeres de toda condición, ignoraban las formas de comunicación escritural no obstante que sus vidas eran parcialmente gobernadas por ordenanzas, cédulas reales, sentencias, decretos, resoluciones y demás textos jurídicos producidos por la Corona. Estas normas escritas afectaban sensiblemente la cotidianidad, obligando a las personas a conocer la ley y a recurrir a los medios escriturales para tratar de mitigar los efectos de las decisiones legales del Reino. Para Zambrano, la mayoría de las cartas nunca llegaron a su destino, pero al igual que todas las expresiones documentales de la ley, eran celosamente guardadas por la Corona en un archivo cuidadosamente dispuesto como memoria histórica que cubría casi todos los aspectos de la vida cotidiana. De esa forma, se constituía la historia de la conformación del imperio, una historia legal y documentada, aunque no necesariamente verdadera, o en rigor, verdadera en tanto verdad documental.

Zambrano argumenta que existe una estrecha relación entre las escrituras marcadas por la impronta de la ley y otros sistemas de notación y comunicación desplegados desde esa época que a su vez determinaron la manera como se produjeron otras formas escriturales, incluyendo las no institucionales, y adicionalmente, cómo las "prácticas históricas, sociales y políticas de alfabetización, conformaron un locus crucial de la cultura colonial porque el discurso legal gobernó un régimen de verdad”. (Zambrano, 2000, p. 167).

Si trasladamos el planteamiento del mecanismo escritural propuesto por Zambrano a las condiciones de trabajo del sector público, encontramos que hay algunas semejanzas relevantes para nuestro propio análisis, en particular, aquellas que nos permiten entender la forma como la vida de una persona que labora en una entidad pública pretende ser documentada y legalmente gobernada bajo la lógica managerial promovida desde organismos internacionales como la OCDE. Así como sucedía en el periodo analizado por Zambrano, actualmente se pretende gobernar la vida de las personas en las entidades a través de las prescripciones contenidas en los documentos institucionales que incorporan la lógica managerial.

El discurso legal desarrollado en la Ley 909 en la CIFP, así como en otros documentos institucionales que serán retomados en el siguiente apartado, constituyen un régimen de verdad sobre la vocación del servicio. Colombia es un país en cuya tradición jurídica legalista se buscan resolver problemas como el de la gestión de personal en el sector público expidiendo una ley o generando un documento de política pública, y así sucede al parecer desde la Colonia. Sin embargo, las personas impugnan, apropian o resignifican los mandatos previstos en los documentos. Como veremos a continuación, la imposición de la lógica managerial no sólo es un mecanismo de gobierno promovido desde el exterior como una forma de colonialidad epistémica que logra imponerse sin resistencia alguna; paradójicamente, esta nueva forma de intervención colonial pone en evidencia las tensiones, conflictos y subjetividades que ha provocado la flexibilización laboral neoliberal.

\section{Análisis de prácticas institucionales con apoyo en las perspectivas críticas}

En la última década se han dado cambios en las formas de organización y administración del trabajo dentro de las entidades públicas. Como se explicó, muchas prácticas y conceptos propios de la gestión de la empresa privada han colonizado los espacios del empleo público. Herramientas de gestión, procedimientos, indicadores, instrumentos y demás técnicas provenientes de la administración de empresas, han sido implementadas en las entidades estatales.

Para comprender cómo ha ocurrido este cambio, algunas investigaciones que parten de la perspectiva crítica se han concentrado en "seguir los instrumentos", investigaciones conocidas como etnografías de los dispositivos (Sisto \& Zelaya, 2013). Otras investigaciones se han enfocado en las transformaciones de la subjetividad que estos trasplantes suscitan. De acuerdo con Sisto y Zelaya, esta segunda variante de la literatura 
explora las transformaciones ocurridas a través de herramientas cualitativas, como etnografías del espacio laboral, análisis de narrativas identitarias y estudios críticos del discurso.

Nuestra investigación combinó los enfoques descritos por Sisto y Zelaya, en tanto identificó los elementos que conforman la experiencia cotidiana de los empleados, expresada en la manera como ellos se relacionan con los documentos institucionales a través de los cuales se configuran los discursos oficiales sobre las competencias, perfiles, funciones, tareas, aptitudes, destrezas y demás aspectos que integran la función pública dentro de la cual se prescribe la vocación del servicio en un ambiente marcado por la flexibilización laboral.

Así, identificamos las tensiones en función de su carácter emergente en las tres fuentes de información: entrevistas, diarios de campo y documentos. Toda la información recogida se analizó como contrapunto de la teoría producida a propósito de la vocación del servicio. A continuación presentaré dos de las discusiones que se derivaron del análisis hecho por el equipo de investigación.

\section{Tensiones entre los funcionarios provocadas por la flexibilización laboral}

Para ingresar al servicio público la regla general es la carrera administrativa. No obstante, las entidades del Estado recurren con frecuencia a la contratación por prestación de servicios para realizar tareas misionales. Entre una y otra forma de vinculación existen diferencias legales importantes: los funcionarios ingresan al servicio público a través del sistema de carrera administrativa, gozan de estabilidad laboral y de las prestaciones sociales propios de una vinculación laboral; en tanto que los contratistas no cuenta con ninguno de esos derechos, entre otras diferencias importantes. Esto genera tensión entre los funcionarios de carrera y los contratistas. Los primeros ven a los segundos como cuotas clientelistas, personas que ingresan a la administración porque tiene un padrino político; los contratistas ven a los de carrera como personas atornilladas a sus puestos, burócratas que no trabajan porque tienen garantizada su estabilidad. Estos prejuicios generan mutua desconfianza como se advirtió a través de la observación participante:

Yo pienso que hay muchos funcionarios de carrera que no desarrollan una buena labor, pero como tienen ese status de CARRERA, es decir que a pesar de que no realicen sus funciones no pueden ser despedidos, les están quitando la oportunidad a otra persona, quizá contratista, de desenvolverse y realizar un trabajo de manera eficiente. (Diario de campo agosto de 2012)

La representación que pesa sobre los funcionarios de carrera, como sujetos abúlicos que privan de oportunidades laborales a otros, oscurece aspectos importantes del sistema de carrera administrativa: como el hecho de ser una herramienta en contra del clientelismo y la desigualdad. Los funcionarios de carrera cargan con el estigma de ser los residentes de la zona de confort, un estereotipo que es posible gracias a los prejuicios: atributos supuestamente asignados al sujeto y naturalizados a través del lenguaje, mediante la repetición continua e incesante del mensaje cuyo contenido termina por constituirse en una parte del universo de referencia de los hablantes. Interlocutores e intérpretes, emisores y receptores del mensaje, asumen una propiedad que le es atribuida al empleado de carrera, naturalizando así un lugar para ellos dentro del servicio público. Una vez naturalizado ese lugar, negar la necesidad del sistema de carrera para imponer formas flexibles de trabajo, como los contratos de prestación de servicio, resultan consecuencias obvias del management aplicado a la administración pública.

La flexibilización laboral que promueve el discurso managerialista, precariza las condiciones de trabajo de quienes laboran para el Estado y aumenta la tensión entre ellas, como se advierte al comparar la situación de los contratistas frente a los empleados de carrera administrativa; esto señalaba una estudiante en su práctica de observación dentro del DAFP:

Situaciones como las que suceden con las personas que son contratistas y que bajo lo que está estipulado no deben cumplir con un horario laboral (...), mas se presenta todo lo contrario: cumplen un horario laboral como el de los demás pero sus prestaciones y beneficios no son proporcionales a lo que trabajan, lo cual en mi opinión es excluyente, injusto y desconcertante, pues ver cómo otras personas que tiene asegurados sus puestos, como los de carrera, los cuales parece ser que aunque no 
rindan, no cumplan metas y demás, no pueden ser despedidos y tienen como un régimen especial, lo cual es un constructo que se maneja y se ha naturalizado en esta entidad, perpetuando el estado de confort del empleado y la idea de que el empleado de carrera es "intocable". (Diario de campo agosto de 2012)

La subjetividad laboral es un producto en permanente elaboración, en el cual, el lenguaje de la psicología organizacional y del management, concurren en la elaboración de estereotipos a través de los cuales se asignan roles y funciones. El discurso crea los lugares desde los cuales el sujeto puede ser, al mismo tiempo y de forma binaria, disciplinado y analizado, intervenido y regulado, estereotipado entre dos extremos: agente de cambios con vocación del servicio o anquilosado residente de la zona de confort sin vocación del servicio.

$\mathrm{Al}$ empleado de carrera administrativa suele asociársele con una categoría propagada por la psicología organizacional: la zona de confort. Mediante esta etiqueta, se describe la situación del funcionario anclado al puesto de trabajo, a la seguridad de su manual de funciones, por ende renuente al cambio. La psicología asocia este comportamiento con la imagen negativa de un funcionario que entorpece las dinámicas de transformación dentro de la entidad, contagiando con su actitud a los demás trabajadores. Se trata de una disfuncionalidad organizacional susceptible de intervención mediante el saber profesional, es decir, una patología que puede ser corregida. Esto consignaba una estudiante durante su observación en la entidad:

Me llama mucho la atención que desde que yo llegué a la entidad los servidores se quejan constantemente porque todo lo que se realiza en gestión humana es muy plano y no hay nada que genere impacto, pero cuando se proponen iniciativas nuevas que en cierta forma generan un impacto en la cultura organizacional, la gente no responde de la mejor manera, pareciera que le temen al cambio, le temen a arriesgarse a apoyar iniciativas nuevas porque están en una zona de confort, de estabilidad en la que así estén "inconformes" no quieren cambiar; de pronto se sienten acosados a tener que adaptarse de nuevo a situaciones que les van a implicar un cambio personal. (Diarios de campo del 13 al 17 de agosto de 2012)

Expresiones como zona de confort, resistencia y temor al cambio, se emplean como muletillas a través de las cuales se realiza una doble función a través del discurso: por un lado, este se legitima, pues pese a su reducida o incluso nula capacidad explicativa, tales expresiones emitidas por el sujeto experto titular de un saber científico (administración, psicología o cualquier otra disciplina) envisten de autoridad las prescripciones y diagnósticos emitidos a propósito del comportamiento y la forma de ser de los sujetos observados; simultáneamente, se reafirma la condición del sujeto observado como alguien que debe ser disciplinado, en esta caso: motivado para que tenga vocación y desempeñe con ánimo sus funciones. Este es un procedimiento propio de la colonialidad epistémica: el sujeto-objeto de intervención no sabe, no tiene consciencia de lo que es bueno para él, por ende, debe ser corregido, enseñado, instruido, disciplinado. La dualidad salvaje civilizado, subdesarrollado desarrollado, cobra vida nuevamente en la retórica organizacional.

Sin embargo, a lo largo de la práctica otras estudiantes lograban cuestionar algunos lugares comunes construidos por el lenguaje que fusiona administración y psicología:

Por otro lado, es importante mencionar cómo en la literatura se pretende crear y hasta llegar a naturalizar un trabajador proactivo que salga de su zona de confort, sea libre y feliz con las decisiones que él mismo "cree que toma" y que por lo que se ha evidenciado en el área es completamente opuesto de lo que dice la academia y lo que muestra la práctica. Lo anterior, teniendo en cuenta que por lo menos en el ámbito público el trabajador que lleva años desempeñándose en las mismas labores se siente más seguro teniendo el control de los temas que desarrolla, aunque esto no quiere decir que necesariamente esté feliz con la retribución monetaria y la valoración que se le da a su trabajo, y que por tanto genera una inconformidad que puede llegar a ser aminorada por un ascenso o cambio de dependencia, mientras esta tenga que ver en algún punto con el trabajo que desempeñó en el pasado o en cierta medida con temas que conecten su nuevo trabajo con el anterior. (Diarios de campo de octubre de 2012)

Los mecanismos mediante los cuales un funcionario de carrera valora la certidumbre que le ofrece la estabilidad laboral pueden provocar una lectura negativa de sus condiciones legales como empleado de carrera. Por ejemplo, en el trabajo etnográfico aparecieron de manera recurrente afirmaciones de los funcionarios de dirección sobre la ineficacia del sistema de evaluación: "es complejo ver cómo en la organización las personas que tienen ese estado (en referencia a la estabilidad de los empleados de carrera) se pasan con sus privilegios 
pues se sienten en su zona de confort y como saben que no los pueden sacar del trabajo tan fácil, pues hacen las cosas de la peor manera; ya lo he visto en otras partes del departamento." (Diario de campo del 24 al 27 de julio de 2012). Y en otro reporte de campo se encuentra lo siguiente: "afirman los jefes de talento humano: «uno como jefe, por no tener problemas con los funcionarios, prefiere ponerles a los más problemáticos pero que no son los que más trabajan- el 100\% en la evaluación y así ahorrarse los reclamos»” (Diarios de campo del 27 al 31 de agosto de 2012).

Otras son las problemáticas de los contratistas, quienes -se supone- prestan su servicio de manera independiente a las entidades públicas en tareas puntuales, ocasionales y que no pueden ser realizadas por las entidades públicas mediante el personal de planta. La vinculación de los contratistas debe ser temporal y sin relación de subordinación, pero en la práctica organizacional no es así. La relación de los contratistas dentro de las entidades públicas transcurre como la de cualquier funcionario de planta: cumpliendo horarios, obedeciendo órdenes y ejecutando labores no previstas en el contrato. En pocas palabras, los contratistas se encuentran con frecuencia en situaciones de evidente subordinación, lo que genera conflictos:

Cuando se empezó a construir y pensar en esta sesión (sesión en la que se otorgarían incentivos por desempeño adelantada por
el área de bienestar), en un inicio se había pensado en que todos iban a participar (contratistas, pasantes y de planta) por ello se
habían tenido en cuenta en los refrigerios, chocolates y encuestas de valores (donde podían nominar a contratistas) pero fue
sorpresivo cuando llegamos el miércoles y nos dijo mi jefe que por orden de la directora los contratistas no podían participar
en esta sesión. Por ello, me tocó enviar un correo a los miembros del comité de valores contándoles lo sucedido y avisándoles
que se iba a pasar devolviendo los $\$ 1.000$ de los contratistas que habían aportado para llevar a cabo esta celebración. Mi
pregunta a ello fue epor qué hay cosas en las que participan de bienestar pero en otras no? El abogado del área, me explicó
que por ley solo los funcionarios de planta pueden participar en los programas de bienestar social. Esto generó un malestar
muy grande entre las personas. Al devolver la plata recibí comentarios como "esto es discriminación", "meparece horrible que
nos saquen de esto así", "Gestión humana está cometiendo un grave error", etc., lo cual es definitivamente comprensible. Lo que
yo percibí frente a ello, es que como la sesión de incentivos es algo que aplica meramente a los empleados de planta y que
esto está cobijado bajo un decreto, ahí si se debe cumplir, entonces, ellos actúan por conveniencia, cuando la ley no los puede
"pillar" pues los contratistas pueden participar, pero cuando es algo que puede ser auditado por la importancia que tiene en
la ley ahí si los excluyen. (Diario de campo del 24 al 28 de septiembre de 2012)

La discriminación, sumada a la desprotección que el contratista afronta al carecer de estabilidad y de prestaciones sociales, genera malestar entre las personas vinculadas a la administración mediante un contrato de prestación de servicios y entre sus compañeros de trabajo.

$\mathrm{Al}$ excluir a los contratistas de las actividades generadas como parte del bienestar que se le brinda a quienes laboran en la entidad, se les margina de los beneficios y se les niega un lugar en la entidad. De esta manera lo describe un estudiante en el diario de campo:

pienso que este tipo de recordatorios a los contratistas buscan naturalizar la situación de exclusión y de hacer perpetuar este tipo de conductas en el departamento, cuando ya de por sí el funcionario tiene claro que él no está incluido y que esto representa una falta terrible pues pareciese que estos funcionarios se encuentran en un no lugar, ni aquí ni allá, están únicamente cuando de cumplir objetivos y desarrollar trabajo se trata, pero para cuando es de garantizar y brindar bienestar se trata, no. (Diario de campo No 8)

La forma de vinculación laboral utilizada en las entidades públicas genera incertidumbre, lo que dificulta la construcción de una subjetividad que no sea la impuesta por el estereotipo. En medio de esa tensión, la vocación de servicio es construida de formas muy diferentes. Para los contratistas asumir el discurso de la vocación de servicio que los documentos y los directivos de las entidades promueven, es una cuestión de supervivencia: asumir las condiciones que les sean impuestas sin importar el horario, la carga o el tipo de funciones a realizar, es tener vocación. Ellos asumen lo peor de la flexibilización laboral, pues de lo contrario la renovación estará en riesgo.

En cambio, para el funcionario de carrera la vocación del servicio tiene que ver con el compromiso de cumplir a cabalidad lo establecido en su manual de funciones de manera ética y precisa. No hacerlo puede significar el riesgo de afrontar un proceso disciplinario por extralimitación de funciones, dado que por norma 
legal los empleados públicos sólo pueden hacer aquello que está expresamente consagrado en su manual de funciones. Esto genera una tensión adicional: el discurso del management exige al servidor público ser proactivo, innovador y creativo, sin tener en cuenta su forma de vinculación con la entidad. Esta lógica choca con el empleo público, que por definición legal es reglado y sujeto a las competencias asignadas por el manual de funciones.

El cuestionamiento por la vocación del servicio debe plantearse de manera diferenciada ente los funcionarios de carrera y los contratistas, pero además, es un asunto que amerita ser ubicado en el contexto más amplio emergente en la tensión de tres vectores: los derechos fundamentales en el trabajo, la tendencia impuesta por el management sobre la política de empleo público en Colombia, y finalmente, la necesidad de reestructuración periódica del Estado ante las transformaciones culturales y técnicas que influyen en la administración pública.

\section{La relación entre la estabilidad, la movilidad y la flexibilidad}

El derecho a la estabilidad laboral del servidor público es fundamental para el cumplimiento de los fines previstos en la administración, pues tal garantía combate el nepotismo y el clientelismo, la arbitrariedad y la desviación de poder. Garantizar la estabilidad laboral permite mantener los intereses superiores del Estado y la continuidad de las políticas públicas al margen de los cambios de gobierno y los intereses partidistas. Adicionalmente, la estabilidad es una herramienta eficaz para atraer al servicio público a personas cualificadas, a la vez que les permite proyectar sus decisiones de manera independiente pero ceñidas a la ley, anteponer las prioridades de la planeación y de lo público a los intereses del gobierno de turno; y en lo personal: construir relaciones de pertenencia, edificar un proyecto de vida y proyectar su realización profesional.

El derecho a la estabilidad es un principio fundamental en el trabajo. En el caso de los servidores públicos, la estabilidad se garantiza a través del sistema de carrera contemplado en el artículo 125 de la Constitución Política. De allí que la regla general sea la vinculación de los funcionarios mediante ese sistema, y solo excepcionalmente, se admiten otras formas de vinculación flexible de acuerdo con necesidades y condiciones concretas previstas por la administración.

Internacionalmente, la estabilidad laboral del empleado público se incluye en la CIFP: "La estabilidad del empleo público y su protección frente a la destitución arbitraria, sin perjuicio de la duración, indefinida o temporal, que se establezca." (CIFP, 2003, epígrafe 7, literal c).

No obstante, la estabilidad laboral es insuficiente si el funcionario carece de estímulos enfocados a evitar el anquilosamiento en el ejercicio del cargo. Al respecto, la Corte Constitucional en la Sentencia C-250 de 2013 señaló que la estabilidad y posibilidad de promoción son requisitos fundamentales para garantizar el derecho al trabajo. La movilidad del servidor depende de las posibilidades reales de ascenso, las cuales lo motivarán a continuar capacitándose y a adquirir nuevas competencias y destrezas. De cumplirse estos cometidos, se configura una situación favorable para el trabajador y se generan beneficios para la administración, al reducir la rotación y aumentar las posibilidades de atraer y retener a los funcionarios más motivados y mejor capacitados.

Mediante el sistema de carrera adoptado en la Ley 909 de 2004, el legislador no fue ajeno a esta exigencia del empleo público, por lo que contempló los "cuadros funcionales de empleo", a través de los cuales se busca garantizar la movilidad vertical y horizontal de los servidores públicos. La primera alude al conjunto de posibilidades de ascenso disponibles en la planta de personal, de manera que los funcionarios accedan a empleos de mayor responsabilidad y mejor salario. La horizontal, se refiere a la posibilidad de moverse dentro de la planta a un empleo de igual responsabilidad y nivel de remuneración, en el que el empleado podrá aprender y desempeñar tareas diferentes a las habituales. En ambos casos, el funcionario puede desarrollar nuevas competencias y habilidades que contribuirán a su crecimiento personal y profesional.

En la CIFP la movilidad se consagra como elemento central para el desarrollo de los fines encargados a la administración pública. La Carta señala que la manera de clasificar los puestos de trabajo debe propiciar 
estructuras ordenadas y "dotadas de la flexibilidad necesaria para facilitar la movilidad funcional y geográfica de las personas por razones de interés personal u organizativo y el reconocimiento de la mejora profesional mediante la asignación de tareas de superior dificultad o responsabilidad." (CIFP, 2003, num. 19).

La flexibilidad es fundamento de la movilidad y oportunidad de mejorar las condiciones objetivas de trabajo. Se trata de garantizar a los servidores públicos la posibilidad de asumir nuevos retos, adquirir otras competencias, conocer diferentes posibilidades de acción, generar cambios que eviten la rutina y la frustración producto de la realización de tareas repetitivas y monótonas. En síntesis, flexibilidad materializada en movilidad o promoción, y no en la reducción de las garantías laborales de los empleados en función de la rentabilidad económica de las entidades.

Ley 909 de 2004 establece: "La flexibilidad en la organización y gestión de la función pública para adecuarse a las necesidades cambiantes de la sociedad, flexibilidad que ha de entenderse sin detrimento de la estabilidad de que trata el artículo 27 de la presente ley." La flexibilidad entendida no como disminución de las garantías laborales, sino como un criterio básico para satisfacer los intereses generales y la efectiva prestación del servicio. Este sentido de flexibilidad es congruente con la CIFP, según la cual la relación flexibilidad-movilidad es un elemento clave en la organización de las labores y en la descripción de los puestos de trabajo. Al respecto, el epígrafe 17 de la CIFP señala que tal descripción debe promover el equilibrio entre la definición precisa de la tarea, de forma que sea posible la especialización en el trabajo, y la flexibilidad como elemento imprescindible para adaptar las tareas a las cambiantes circunstancias.

En el epígrafe 24 de la CIFP, relativo al sistema de acceso al empleo público, la Carta reitera la necesidad de contemplar procesos de movilidad o promoción adecuados para garantizar las adaptaciones requeridas para el acceso al empleo, que en ningún momento contradicen los principios y criterios adoptados por la CIFP. Incluso, la evaluación de rendimiento contemplada en el epígrafe siguiente, se articula a la promoción, en tanto a través de aquella se puede garantizar la obtención de información veraz necesaria para tomar decisiones trascendentales en materia de movilidad. Pero otra es la realidad de las prácticas de gestión de personal en el sector público. La flexibilización laboral practicada en las entidades públicas de Colombia se corresponde con lo planteado por Ludmila Costhek, quien la define como el "conjunto de formas contemporáneas de eliminación de derechos laborales y, más que eso, de transferencia a los trabajadores de los riesgos, los costos y el trabajo no remunerado." (Costhek, 2018, p. 32).

En las prácticas concretas dentro de las entidades públicas, la movilidad riñe con la estabilidad brindada por el sistema de carrera administrativa, lo que suspende la posibilidad de la flexibilidad en el sentido planteado por la CIFP. La movilidad resulta ajena a los funcionarios de carrera, como lo expresaba una funcionaria del Ministerio de Hacienda entrevistada durante la investigación:

Hay mucha rotación en los cargos de libre nombramiento y remoción; podemos evidenciar que personas que simplemente antes de que hayan cumplido un año ya están pasando, ya están subiendo, normalmente lo hacen de a dos grados o a veces puede ser mas, mientras que esa situación en los cargos de carrera administrativa tiene que ser luego de que surja un proceso y la movilidad no es ni tan continua, ni tan rápida como la figura o lo que pasa en libre nombramiento y remoción. (Diario de campo del 23 de septiembre de 2013)

La ausencia de movilidad genera frustración para los funcionarios de carrera, pero la falta de estabilidad es un generador de incertidumbre para las personas que no están vinculadas a través de la carrera, como los contratistas. Así, en el sector público, si se goza de estabilidad se carece de movilidad, y si se desea movilidad, es necesario renunciar a la estabilidad. Los hechos contradicen la pretensión de los documentos, generando una tensión entre el ideal normativo que regula la función del servidor público y la realidad impuesta por las prácticas dentro de las entidades. En medio de estas tensiones se construyen subjetividades complejas y contradictoras que no pueden ser entendidas sin tener en cuenta el marco más amplio de imposición de lógicas propias de la administración de empresas en la gestión de lo público.

De esa forma se experimenta aquello que con base en Zambrano denominaba como una judicalización de la vida cotidiana en la entidad pública, donde lo único que parece importar es el cumplimiento de las formas 
por las formas mismas, los supuestos legales que prescriben una flexibilidad que en la práctica se torna en algo completamente distinto. Como en el mundo colonial descrito por Zambrano, las formas escriturales aun gobiernan la vida de las personas, aunque sus realidades disten mucho de las situaciones buscadas por el documento.

La tensión entre movilidad y estabilidad laboral de los funcionarios públicos detectada en esta investigación fue desarrollado con más detalle en un trabajo publicado por Camilo Pulido y Johanna Burbano (2017), del cual cabe destacar el siguiente fragmento recogido dentro del trabajo de campo:

Lo que vas a ver en muchos cargos de aquí es que hay muchos que han estudiado una carrera, son profesionales pero están en cargos técnicos o asistenciales y no pueden acceder a ser (cargos de) profesionales porque la entidad dice que no tienen la experiencias, entonces ese es otro absurdo de la carrera administrativa (...) aquí tú ves que el de Almacén está de operario y es administrador de empresas... (Pulido \& Burbano, 2017, p. 23)

La vida de quienes trabajan en las entidades públicas es gobernada por el discurso propio del management que se realiza a través de las prescripciones legales contenidas en los documentos institucionales, así como mediante las prácticas de intervención promovidas por disciplinas como la psicología y la administración. Los supuestos normativos e institucionales consolidan un régimen de verdad sobre la vocación del servicio, que se instala con relativa facilidad en un país que, como Colombia, obedece a una tradición legalista. A través de esta tradición se pretende resolver problemas cotidianos como la falta de motivación en el servicio, la abulia de los servidores públicos, la alta rotación en algunos cargos, el anquilosamiento en otros, la falta de reconocimiento de los funcionarios de carrera, la desigualdad, la inequidad, y un largo etcétera; se piensa que expidiendo una ley o generando un documento de política pública, se promoverá la vocación del servicio, se resolverán las tensiones y se hará eficiente la prestación del servicio público. Al mismo tiempo, las reformas legales promueven la precariedad en el trabajo y proliferan las vinculaciones por prestación de servicios. Desde la colonia vivimos, más que en una dualidad entre los mandatos legales y las prácticas, en una neurosis normativa donde la forma prima sobre la experiencia vivida, gobernados por leyes ineficaces para garantizar los derechos pero muy eficaces para promover estereotipos y subjetividades laborales sumisas.

No obstante, las personas en las entidades reflexionan a partir de esta dualidad para impugnar los mandatos previstos en los documentos. Las formas de intervención colonial promovidas por el management, paradójicamente, logran poner en evidencia las tensiones, conflictos y subjetividades que las formas tradicionales de administración pública no hacían más que esconder. La irrupción de la lógica de la empresa privada dentro de las entidades públicas como parte de las imposiciones de los organismos internacionales en países como Colombia, ha exacerbado las tensiones, al promover, en lo legal, la proliferación de los contratos por prestación de servicios, y en la subjetividad, la representación de un servidor de quien se espera que tenga vocación, pero que no exija sus derechos.

\section{Conclusiones}

$\mathrm{Al}$ indagar por la vocación del servicio en el sector público en Colombia es posible detectar la manera como progresivamente se afianzó la lógica de la empresa privada como consecuencia de la adopción de una cultura managerial impuesta desde los organismos internacionales, lo que generó tensiones en las relaciones entre quienes trabajan en las entidades públicas así como cambios importantes en las subjetividades laborales.

Las prácticas y discursos institucionales a través de los cuales se define y se prescribe la vocación del servicio parten de considerar que esta es un atributo natural propio de todas las personas que laboran en la administración pública y que se puede incentivar sin importar las condiciones objetivas de trabajo, no obstante lo cual a través de la investigación se constató que existe una gran distancia entre la manera como se prescribe la vocación y la manera como esta se resignifica por las personas en las condiciones concretas 
del trabajo. La vocación del servicio no es una cualidad inherente de las personas, sino el producto de las relaciones y las condiciones de labor.

A través de las herramientas empleadas en la investigación percibimos que la lectura de los documentos no bastaba para dar cuenta de la compleja red de relaciones a través de las cuales se instala la cultura managerial en el empleo público, por lo cual acudimos a la observación de la cotidianidad en las entidades. Encontramos que hay un flujo constante entre el lenguaje documental y las prácticas cotidianas de los funcionarios públicos. Las personas no son sujetos pasivos de las órdenes, directrices y prescripciones que se imparten a través de los documentos, ellas resignifican el lenguaje a través de sus propias discursividades. Allí, como señalan Das y Poole (2008), el Estado se torna intermitente, en tanto los documentos que expide están sujetos a permanentes interpretaciones y cambios de sentido en las prácticas de los mismos funcionarios.

Estas reflexiones y marcos teóricos de partida, nos llevaron a la necesidad de problematizar la vocación del servicio en contextos marcados por la colonialidad del saber técnico que se introduce en las entidades públicas. El lugar de producción del discurso resulta difuso, pues bien puede ser la OCDE, el BID o alguna otra organización internacional. Desde allí, los conceptos inician su respectivo viaje hasta las agendas públicas de los gobiernos como el de Colombia.

La política del "buen gobierno", como se planteó en el Plan de Desarrollo del gobierno Santos, acogió sin reparo las directrices que los organismos internacionales le dictaron (y le siguen dictando) a países que dócilmente acatan las órdenes. Modernizar el estado implicaba hacer reformas, pero sobre todo, construir una subjetividad del servidor público sin convicciones propias ni de clase. Las convicciones del nuevo servidor público serán las que dicte el manejo "técnico" de lo público.

Tal y como le sucede a Obi Okonkwo, en la novela Me alegraría de otra muerte, tanto nuestros funcionarios de carrera, como nuestros contratistas, pueden devenir en sujetos a la deriva a causa de las condiciones impuestas por las restructuraciones de las entidades públicas. Esas reestructuraciones atienden a los mandatos de los organismos internacionales y a los presupuestos de la lógica managerial, pasando por alto las tensiones cotidianas que se ocasionan, entre otras razones, por la inequidad de las formas de vinculación al sector público. Las consecuencias de la flexibilización, en virtud de la cual las entidades estatales buscan disfrazar verdaderas relaciones laborales mediante los contratos de prestación de servicios, las asumen las personas que trabajan en el sector público, quienes se confrontan diariamente al advertir las desigualdades que la administración promueve.

Aquí se encuentra uno de los rasgos coloniales del discurso de la vocación del servicio: el que construye un tipo de empleado de quien se espera que obre guiado por la "ética del desinterés" independiente de sus condiciones de trabajo. Como le sucede a Obi Okonkwo, antihéroe protagonista de Me alegraría de otra muerte, nuestros servidores públicos son representados en los discursos institucionales como sujetos carentes de convicciones propias, orientados por un interés individual: mantenerse en la administración o en su zona de confort. Este hecho omite las condiciones estructurales tanto como los efectos del discurso, elementos que en este artículo tan solo alcanzamos a atisbar.

\section{Agradecimientos}

Agradezco a Camilo Pulido, Johanna Burbano y Luz Mery Carvajal, con quienes compartí el espacio de coordinación durante esos años y discutimos varias de las ideas planteadas en este artículo.

\section{Referencias}

Agüero, J. (2010). Michel Foucault y la gubernamentalidad financiera. Reflexiones sobre la crisis financiera internacional. Visión de Futuro, año 7, 14(2). 
Ascorra, P. (2010). Aportes de las ciencias sociales al Nuevo Management. Psicoperspectivas, 9(1), 1-6. Doi: 10.5027/ psicoperspectivas-Vol9-Issue1-fulltext-119

Aubert, N., \& Gaulejac, V. (1993). El coste de la excelencia. Barcelona, Paidos.

Barrascout, E. (2012). Vital Resource. The only element that emerges strong and irreplaceable for any company, despite technology and globalization. Thesis. Atlantic International University Honolulu, Hawai.

CEPAL (s.f.). Documento Visión Colombia II Centenario: 2019. Disponible en línea https://archivo.cepal.org/pdfs /GuiaProspectiva/visionColombiaIIcentenario_2019comple.pdf

Chandler, J., Barry, J., \& Clark, H. (2002). Stressing academe: The wear and tear of the new public management. Human Relations, 55(9), 1051-1069. https://doi.org/10.1177\%2F0018726702055009019

Costhek, L. (2018). Uberización: nuevas formas de control, organización y gestión del trabajo. En O. López \& A. Cuevas (eds.), Trabajo, Derecho y Subjetividad. Bogotá: Universidad Libre.

Dall, H. (2009). New public management, care and struggles about recognition. Critical Social Policy, 29(4), 634-654. https://doi.org/10.1177\%2F0261018309341903

Das, V., \& Poole, D. (2008). El Estado y sus márgenes. Etnografías comparadas. Cuadernos de Antropología Social. Buenos Aíres: UBA.

Foucault, M. (2000). Defender la sociedad. Buenos Aires. Fondo de Cultura Económica.

Foucault, M. (2006). Seguridad, territorio, población. Curso en Collège de France: 1977-1978. Buenos Aires: Fondo de Cultura Económica.

Foucault, M. (2010). El orden del discurso. Barcelona: Tusquets Editores.

Garrido, M. (2017). Las agencias estatales de naturaleza especial en Colombia. Revista Digital de Derecho Administrativo, 17, 243-290 https://doi.org/10.18601/21452946.n17.13

Gómez, C., \& Arango, X. (2012). Administración pública y gobernanza: el papel de la gestión de la ética en las organizaciones públicas. Daena: International Journal of Good Conscience, 7(3), 109-122.

Lira, M. (2009). ¿Cómo puedo mejorar el servicio al cliente? Técnicas para perfeccionar la actitud en el servicio a clientes. Recuperado de: https://comunidadilgo.org/back/_lib/file/doc/portaldoc453_3.pdf

López, P. (2010). Biopolítica, liberalismo y neoliberalismo: acción política y gestión de la vida en el último Foucault. En S. Arribas et al. (coords.), Hacer vivir, dejar morir. Biopolitica y capitalismo (pp. 39-61). Madrid, CSIC/La Catarata.

Muñoz, A. (2006). El empresario de sí mismo: una visión desde la economía. Revista Ciencias Estratégicas, 14(15), 67-82.

Ospina, S., \& Hofmann-Pinilla, A. (2015). Voces y vivencias: la gestión del talento humano vista desde adentro de las organizaciones públicas colombianas. En P. Sanabria (comp.). Gestión estratégica del talento humano en el sectorpúblico: estado del arte, diagnóstico y recomendaciones para el caso colombiano (pp. 119-146). Bogotá: ESAP, Universidad de los Andes, DAFP.

Pareja, B. (s.f.). Ventaja generacional y liderazgo para el cambio. En La Nueva Generación Executive: los directivos de boy que impulsarán el cambio necesario del mañana. Recuperado de: http://static.byhours.com/press_files/cola boraciones/libro-la-nueva-generacion-executive.pdf

Parker, M. (2002). Against management. Londres: Polity Press.

Peña, W. (2012). De la responsabilidad social en la sociedad del riesgo: Más allá de la libertad de ser empresarios de sí mismos. Revista Latinoamericana de Bioética, 12(2), edición 23, 78-89.

Pierbattisti, D. (2008). La privatización de los cuerpos. La construcción de proactividad neoliberal en el ámbito de las telecomunicaciones 1991-2001. Buenos Aires: Prometeo.

Pierbattisti, D. (2012). Managment, subjetividad y cultura política: el nuevo espíritu del capitalismo a veinte años de la privatización de ENTEL. Cuadernos de Relaciones Laborales, 30(2), 459-495.

Pulido, C., \& Burbano J. (2017). De la construcción del servidor público y del lugar de la vocación en las condiciones de trabajo contemporáneas. Temas e Investigaciones en Psicologia Organizacional y del Trabajo. Cali: Editores Erico Renteria y Sigmar Malvezzi. Editorial Universidad del Valle. 
Pulido, C. (2008). Condiciones flexibles de trabajo y conocimiento psicológico: el caso del servicio tradicional de transporte de pasajeros en Bogotá, Colombia. Revista Iberoamericana de Psicología: Ciencia y Tecnología, 1, 9-18.

Rodríguez, E., \& Grillo, F. (2010). Reforma al empleo público en Colombia. En Memorias de la Renovación del Estado 2002-2010. Bogotá: Departamento Nacional de Planeación.

Sanabria, P. (2015). Estado del arte de la gestión estratégica del talento humano en el sector público: teoría y prácticas. En P. Sanabria (comp.), Gestión estratégica del talento humano en el sector público: estado del arte, diagnóstico y recomendaciones para el caso colombiano (pp. 23-59). Bogotá: ESAP, Universidad de los Andes, DAFP.

Sarria, C. (2015). ¿Las agencias son unidades administrativas especiales? Revista Digital de Derecho Administrativo, 13, 237-252. https://doi.org/10.18601/21452946.n13.12

Sisto, V., \& Zelaya, V. (2013). La etnografía de dispositivos como herramienta de análisis y el estudio del managerialismo como práctica local. Revista Universitas Psychologica, 12(4), 1345-1354. Doi: 10.11144/ javeriana.UPSY12-4.edha

Spink, P. (2009). Los psicólogos y las políticas públicas en América Latina: el big mac y los caballos de Troia. Psicoperspectivas: Individuo y Sociedad, 8(2), 12-34. Doi: 10.5027/psicoperspectivas-Vol8-Issue2-fulltext-70

Thomas, R., \& Davies, A. (2005). Theorizing the micro- politics of resistence: New public management and managerial identities in the UK public services. Organization Studies, 26(5), 683-706. https://doi.org/10.1177\%2F01708 40605051821

Weber, M. (1994). La ética protestante y el espiritu del capitalismo. Barcelona: Península.

Wodak, R. (2003). El enfoque histórico del discurso. En R. Wodak \& M. Meyer (comps.), Métodos de análisis crítico del discurso. Barcelona: Editorial Gedisa.

Zambrano, M. (2000). La impronta de la ley: escritura y poder en la cultura colonial. En C. Gnecco \& M. Zambrano (eds.), Memorias hegemónicas, memorias disidentes. El pasado como politica de la historia. Bogotá: ICANH, Universidad del Cauca.

\section{Notas}

[1] Se emplea este verbo pues fue una expresión literal de la directora del DAFP entre 2008 y 2014, cuya formación de grado es la administración de empresas. Durante una reunión que sostuvimos con ella, una expresión que sintetizó su discurso fue la siguiente: "debemos inyectar la vocación de servicio a nuestros funcionarios". Diario de campo, abril de 2012

* Artículo de investigación científica.

\section{Licencia Creative Commons CC BY 4.0}

Sugerencia de citación: López C., O. A. (2019). Vocación del servicio en Colombia y nuevas formas de colonialismo en la lógica managerial. Cuadernos de Administración, 32(58). https://doi.org/10.11144/Jave riana.cao32-58.vscn 